\title{
Impacto de la apolipoproteína A5 en el riesgo cardiovascular. Modulaciones genéticas y ambientales
}

Departamento de Medicin

Preventiva y Salud Pública, Ciencias de la Alimentación, Toxicología y Medicina Legal, Universitat de València y CIBER Fisiopatología de la Obesidad y Nutrición, Instituto de Salud Carlos III. ${ }^{a}$ Doctoranda en Nutrigenómica y Salud Pública.

${ }^{\mathrm{b}}$ Catedrática Universitaria en Epidemiología molecular y Salud Pública.

Recibido el 8 de junio de 2009, aceptado el 11 de diciembre de 2009.

Fuentes de Financiación proyectos CB06/03/035 PI061326 y PI070954

Correspondencia a: Mercedes Sotos Prieto Dpto. de Medicina Preventiva y Salud Pública, Ciencias de la Alimentación, Toxicología y Medicina Legal, Universitat de València

Universidad de Valencia

Avda Blasco Ibáñez, 15 46010 Valencia

Tfno: 963864166

Fax: 963864417

E-mail: Mercedes.Sotos@uv.es
MERCEDES SOTOS-PRIETO ${ }^{a}$, FRANCESC FRANCÉS, DOLORES CORELLA ${ }^{\mathrm{b}}$

\section{Impact of apolipoprotein A5 on cardiovascular risk. Genetic and environmental modulation}

Triglyceride concentrations are an independent risk factor for coronary heart disease. Apolipoprotein A5 gene (APOA5) has an important role determining triglyceride metabolism and it is a potential cardiovascular risk. However the mechanisms for these actions are not well-known. Despite the different allelic frequency of its major polymorphisms in different populations, multiple studies have shown consistent associations between these variants and fasting triglycerides. Variations in the APOA5 gene have also been associated with postprandial triglycerides, as well as with different sizes of lipoproteins and other markers. Moreover, some of the APOA5 gene variants have been associated with ischemic heart disease, stroke, and carotid intima media thickness, although the references on this issue are scanty and contradictory. This may be due to the presence of gene-environment interactions that have been poorly studied until now. Among the few studies that have examined the influence of environmental factors on possible genetic variations, the most important are those that contemplate possible gene-diet interactions. However, the evidence is still scarce and more research is required in the field of nutrigenomics. To understand the impact of this gene on cardiovascular disease, we review the genetic functionality and variability of APOA5, its associations with intermediate and final phenotypes and gene-environment interactions detected.

(Rev Med Chile 2010; 138: 868-880).

Key words: APOA5 protein human; Coronary disease; Triglycerides.
$\mathrm{E}$ 1 valor de la trigliceridemia en la predicción del riesgo cardiovascular, independiente de los principales factores de riesgo (colesterol total (CT), lipoproteínas de baja densidad (LDLC), presión arterial, fumar, etc) ha permanecido confuso durante los últimos años. Sin embargo, la acumulación de evidencia científica sugiere que la elevación de la concentración de triglicéridos (TG) es un factor de riesgo independiente de enfermedad cardiovascular ${ }^{1-4}$. Un meta-análisis de 21 poblaciones basados en estudios prospectivos con un total de 65.863 hombres y 11.089 mujeres en el que se estudió la asociación entre los TG y enfermedad cardiovascular en población general, encontraron que la elevación de $89 \mathrm{mg} / \mathrm{dL}$ en la concentración de TG estaba asociado con un incremento de $12 \%$ del riesgo cardiovascular en hombres y $37 \%$ en mujeres tras ajustar por CT, LDL-C, HDL-C (lipoproteínas de alta densidad), índice de masa corporal (IMC), presión arterial y diabetes 5 . Esta elevación de la concentración de TG está asociada con diversos factores, como son el estilo de vida, otras enfermedades (diabetes mellitus tipo 2, síndrome metabólico, obesidad), medicación, y factores genéticos. Por ello, resulta crucial evaluar, identificar y controlar el efecto 
conjunto de las variables ambientales y genéticas de la enfermedad teniendo en cuenta los distintos factores ambientales que puedan influir y cómo las interacciones gen-dieta influyen en la susceptibilidad individual a presentar marcadores fenotípicos más desfavorables pudiendo la dieta influir en dichos fenotipos agravando o neutralizando la susceptibilidad genética. Aunque son múltiples los genes que se han relacionado con las enfermedades cardiovasculares, el gen de la apolipoproteína A5 (APOA5) ha surgido recientemente como uno de los más relevantes. Dicho gen se ha implicado en el metabolismo de los TG, aunque todavía no se conoce bien su mecanismo de acción, ni la contribución de sus variantes al riesgo cardiovascular. Por ello, nuestro objetivo es realizar una revisión de los principales trabajos que han analizado dicho gen y su asociación con fenotipos intermedios y finales de riesgo cardiovascular.

\section{Descubrimiento del gen de la apolipoproteína A5, estructura, funcionalidad y relaciones con otras apolipoproteínas}

El gen de la APOA5 fue identificado en 2001 por Pennacchio y colaboradores mediante análisis genómicos comparativos de ratón y humano ${ }^{6}$. El gen de la APOA5 está localizado aproximadamente a $30 \mathrm{~Kb}$ de distancia del grupo de genes bien caracterizados APOA1/C3/A4 en el cromosoma 11q23. Consiste en 4 exones que codifican 366 aminoácidos con $71 \%$ de identidad a apoa5 de ratón y $27 \%$ de identidad al gen $A P O A 4$ humano ${ }^{6}$. La expresión del gen de la APOA5 está modulada por varios factores. Es sobreexpresada en respuesta a factores de transcripción que afectan al metabolismo de TG como el PPAR- (Peroxisome proliferator activated receptor alpha $)^{7,8}$, el receptor nuclear y el ROR $(R e-$ ceptor tyrosine kinase-like orphan receptor) 1 y $4^{9} \mathrm{y}$ los ligandos del receptor X del hígado (T0901317) pueden regular la expresión a través de SREBP-1c (Sterol Element Binding Protein 1C) ${ }^{10}$.

La proteína para la que codifica, apolipoproteína A5 (APOA5), está presente en bajas concentraciones en suero humano (rango de 25 a $400 \mu \mathrm{g} / \mathrm{L}$ ), lo que dificulta su medición en plasma mediante técnicas de inmunoanálisis, y desde su descubrimiento, ha sido asociada con hipertrigliceridemia $^{6,11}$. Está fundamentalmente expresada en hígado donde se secreta a la circulación ligada a las HDL, lipoproteínas de muy baja densidad
(VLDL) y partículas de quilomicrones ${ }^{6,12,13}$. La maduración de esta proteína ocurre por pérdida de 23 aminoácidos correspondientes al péptido señal ${ }^{14}$. En cuanto a su estructura, destaca un importante componente alfa hélice en un contexto de predominio hidrofóbico ${ }^{15}$ donde el extremo C-terminal tiene un papel importante en la unión de la proteína a los lípidos ${ }^{16}$. La creación de modelos knock-out y transgénicos para el gen APOA5 en roedores, así como el doble transgénico y knock-out para APOA5-APOC3 mostró que la apoa5 y la apoc3 podrían jugar un papel opuesto en la regulación de los niveles de lípidos, principalmente los TG. Algunos estudios indican que la APOA5 reduce la concentración sérica de TG por estimulación de liproteínlipasa (LPL) y por inhibición de la producción de VLDL-TG en hígado ${ }^{13,17,18}$. Así, se ha sugerido que APOA5 juega un papel importante en la hidrólisis de los componentes lipídicos de las lipoproteínas, siendo esta proteína clave en la unión a los proteoglicanos de membrana plasmática de la célula endotelial, permitiendo así la actividad lipolítica endotelial sobre las VLDLs, merced a que esta unión mediada por APOA5 mantiene las VLDL en vecindad a la LPL endotelial ${ }^{18}$.

\section{Asociaciones de los principales polimorfismos del gen de la apolipoproteína A5 con distintos fenotipos intermedios de riesgo cardiovascular}

Se han identificado más de 15 polimorfismos de un solo nucleótido (SNPs), es decir, variaciones en la secuencia de ADN que afecta a una sola base y que tienen una frecuencia al menos de un $1 \%$ en la población, en dicho gen. Varios estudios indican una fuerte asociación entre los polimorfismos más comunes (Val53 > Met, -12238T > C, $-1131 \mathrm{~T}>\mathrm{C}$, IVS $3+476 \mathrm{G}>\mathrm{A},-3>\mathrm{G}, 56 \mathrm{C}>$ G (o S19W), 442C > T, 553G > T, Ala315 > Val, $1259 \mathrm{~T}>\mathrm{C}$ ) de la APOA5 y la concentración de lípidos en plasma ${ }^{6,19-22}$. También se ha evidenciado que muchos de ellos se heredan conjuntamente formando bloques haplotípicos, distinguiéndose dos haplotipos independientes identificados por los tag SNPs o SNPs indicadores denominados $-1131 \mathrm{C}>\mathrm{T}\left(A P O A 5^{\star} 2\right)$ y $\mathrm{S} 19 \mathrm{~W}\left(A P O A 5^{\star} 3\right)$ donde tienen un efecto diana en el aumento de la concentración de $\mathrm{TG}^{21,23}$. 
En la Tabla 1 se presentan las frecuencias alélicas obtenidas del alelo menos común de algunos de los polimorfismos más relevantes en distintas poblaciones.

Las asociaciones de estas variantes genéticas con fenotipos intermedios de riesgo cardiovascular obtenidas en algunos estudios, las características de la muestra, así como la frecuencia alélica de los diferentes polimorfismos en cada grupo estudiado se presentan en la Tabla 2, si bien destaca la asociación de los polimorfismos del gen $A P O A 5$ y las concentraciones plasmáticas de TG en ayunas.

Dada la relevancia del estudio postprandial, no solo es importante conocer el efecto de las variantes en el gen APOA5 en ayunas sino tras la ingesta. Se han realizado varios estudios centrados en este aspecto. En la Tabla 3 se muestran los principales ensayos clínicos que estudian la asociación entre variantes genéticas en $A P O A 5$ y los TG postprandiales y los fenotipos intermedios de riesgo cardiovascular.

\section{Polimorfismo -1131T > Cy su asociación con parámetros lipídicos}

La variante del gen de la APOA5 más estudiada es la-1131T > C (también denominada en algunos estudios SNP3). Su asociación con la concentra- ción de TG muestra bastante consistencia y ha sido confirmada en varios estudios que incluían Caucásicos $^{24,}$, Japoneses ${ }^{25}$, Chinos $^{26}$ y Taiwaneses ${ }^{27}$. El impacto de este polimorfismo $(-1131 \mathrm{~T}>\mathrm{C})$ fue más pronunciado en hispánicos que en caucásicos $y$ no fue detectable en afroamericanos ${ }^{21}$.

Se ha propuesto que este polimorfismo podría modular la actividad trascripcional de la proteína ${ }^{28}$ pero este hecho no se ha podido demostrar ${ }^{29}$ llegando a sugerirse que $-1131 \mathrm{~T}>\mathrm{C}$ y el resto de polimorfismos integrantes de haplotipos $A P O A 5^{\star} 2$ carecen de funcionalidad y que las asociaciones encontradas, reflejarían el hecho de que -1131 T $>\mathrm{C}$ es un marcador de variaciones funcionales del gen $A P O C 3^{30}$ siendo los efectos de $A P O A 5^{\star} 2$ debidos al desequilibrio de ligamiento de $-1131 \mathrm{~T}$ $>\mathrm{C}$ con $482 \mathrm{~T}>\mathrm{C}(\text { APOC } 3)^{30}$.

En relación a lo anterior también se ha afirmado que los portadores del alelo -1131C tenían mayor riesgo genético de hipertrigliceridemia ${ }^{25}$.

\section{Polimorfismo S19W y su asociación con parámetros lipídicos}

Son varios los estudios que han demostrado la asociación independiente de este polimorfismo y la hipertrigliceridemia ${ }^{6}$. Este polimorfismo está en una región codificante de APOA5 y resulta en un

Tabla 1. Frecuencia alélica del alelo menos común de algunos polimorfismos del gen de la APOA5 en diferentes poblaciones

\begin{tabular}{|c|c|c|c|c|c|c|}
\hline \multirow[t]{2}{*}{ Variante } & \multirow[t]{2}{*}{$\begin{array}{c}\text { Otra } \\
\text { denominación }\end{array}$} & \multirow{2}{*}{$\begin{array}{l}\text { Posición en el } \\
\text { cromosoma } 11^{\text {a }} \text { y } \\
\text { localización }\end{array}$} & \multirow[t]{2}{*}{$\begin{array}{l}\text { Cambio de } \\
\text { aminoácido }\end{array}$} & \multicolumn{3}{|c|}{$\begin{array}{l}\text { Frecuencia alélica del alelo } \\
\text { rarob }\end{array}$} \\
\hline & & & & Europea & Africana & $\begin{array}{c}\text { Asiática } \\
\text { (CHB) }\end{array}$ \\
\hline rs2075291 & c.553G $>\mathrm{T}$ & $\begin{array}{l}116166602 \\
\text { Exon } 4\end{array}$ & Gly185Cys & 0,008 & 0 & 0,022 \\
\hline rs3135507 & c. $457 G>A$ & 116166698 & Val153Met & 0,06 & b & 0,103 \\
\hline rs662799 & $-1131 \mathrm{~T}>\mathrm{C}$ & $\begin{array}{l}\text { 116168917, región } \\
\text { promotora }\end{array}$ & & 0,017 & 0,133 & 0,267 \\
\hline rs3135506 & c. $56 C>$ G & $\begin{array}{l}\text { 116167617, péptido } \\
\text { señal }\end{array}$ & Ser19Trp & 0,058 & 0 & 0,050 \\
\hline rs651821 & $-3 G>A$ & $\begin{array}{l}\text { 116167789, secuencia } \\
\text { Kozak }\end{array}$ & & 0,017 & 0,195 & 0,267 \\
\hline rs41389248 & IVS3+476G > A & 116167444, Intrón 3 & & 0,031 & b & 0,187 \\
\hline b & c. $944 C>T$ & Exon 4 & Ala315Val & 0,003 & b & b \\
\hline rs2266788 & $1259 \mathrm{~T}>\mathrm{C}$ & 116165896, 3ÚTR & & 0,042 & 0 & 0,244 \\
\hline
\end{tabular}

aPosición de acuerdo a la base de datos de Ensemble. 'Datos no encontrados. 


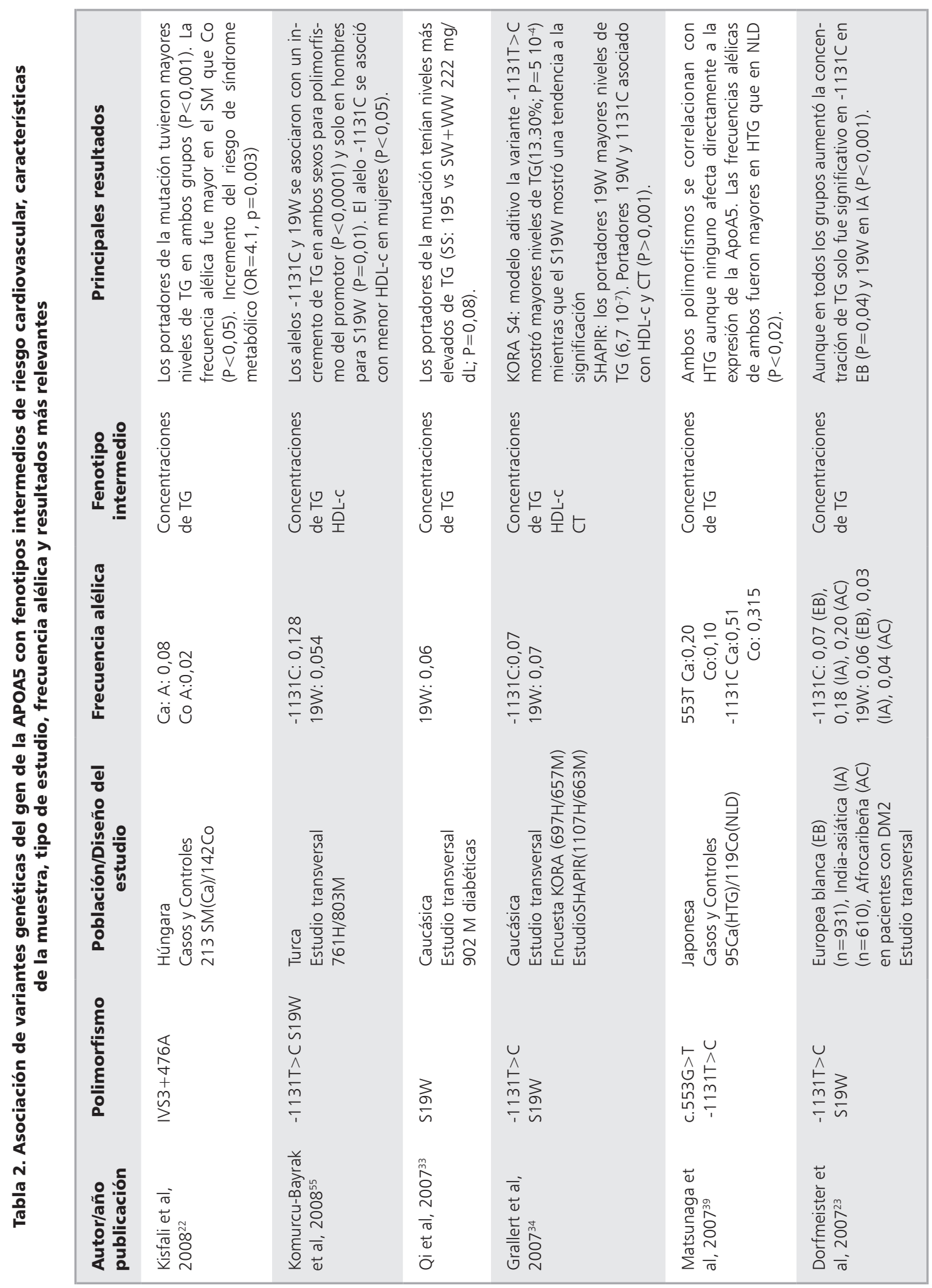









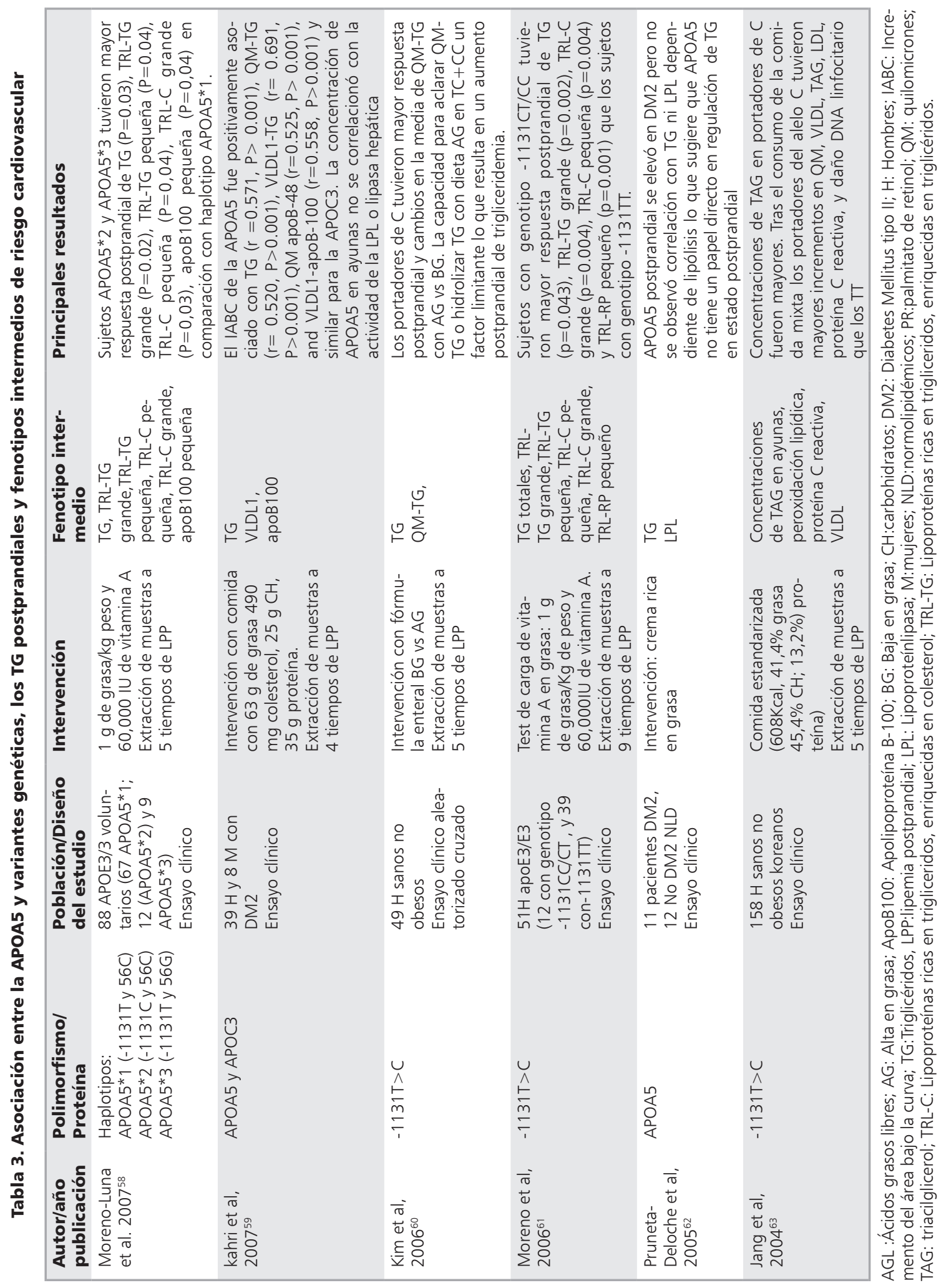


cambio de aminoácido, de serina (Ser) por triptófano (Trp). Es conocido que mutaciones en esta molécula pueden alterar la capacidad de unión de los receptores de las lipoproteínas al producirse un cambio hidrofílico de Ser por el hidrófobo Trp en el péptido señal ya que puede inducir alteraciones en la estructura secundaria de la proteína con su correspondiente impacto en la estructura terciaria, mediante el aumento de la probabilidad en las formas 19W que los residuos 14-16 generaran un alfa hélice. Esta alteración estructural alteraría la capacidad de la proteína, bien por afectación de la inserción de la proteína naciente en el retículo endoplasmático rugoso, bien por alteración de la separación del péptido señal, incluso por conferir en caso de los portadores de la variante 19W una incapacidad para la interacción con los receptores lipídicos ${ }^{29}$.

La variante S19W de APOA5 confiere un mayor riesgo para la hiperlipidemia, específicamente en estado de homocigosis ${ }^{6,31}$. Diferentes estudios confirman que los portadores de la variante $19 \mathrm{~W}$ presentan cifras más elevadas de $\mathrm{TG}^{6,21,29,32,33}$. En el aspecto racial, estudios llevados a cabo en caucásicos también muestran resultados similares ${ }^{31,34}$. Sin embargo, estudios en población canadiense ${ }^{35} \mathrm{y}$ en indios $\mathrm{Pima}^{31}$, no replicaron estos hallazgos, posiblemente debido, en este último caso, a la menor frecuencia alélica de este polimorfismo.

\section{Polimorfismo c.553G > T y parámetros lipídicos}

La mayoría de los estudios de este polimorfismo están basados en población China. De hecho, esta variante no se ha encontrado en caucásicos lo que sugiere la posibilidad de que su presencia sea específica de la raza asiática. En cuanto al mecanismo de acción de este polimorfismo se ha sugerido que podría alterar la formación/ estabilidad de la estructura helicoidal de APOA5, modificando su capacidad de unión a interfaces lipídicas y así alterando la formación de las VLDL $^{37}$.

Este polimorfismo consiste en un cambio de aminoácido de glicina (Gly) por cisteína (Cys) y la asociación más importante con fenotipos intermedios de riesgo cardiovascular encontrada ha sido con la concentración de $\mathrm{TG}^{27,28,37-39}$. No obstante, se ha asociado también con menores concentraciones de HDL-C ${ }^{27,28}$.

\section{Otros polimorfismos $y$ sus asociaciones principales}

En relación a otros polimorfismos de APOA5 la bibliografía al respecto es escasa. Si bien, la variante Val153 > Met no se ha asociado con parámetros lipídicos en un estudio llevado a cabo en población china $^{19}$, en europeos caucásicos se ha encontrado una asociación específica de sexo entre la presencia del alelo Met153 y menores niveles de HDL- en mujeres $^{40}$.

La variante Ala315 > Val no mostró un importante impacto sobre los niveles de TG en un estudio realizado en 3202 individuos y 95 individuos con hipertrigliceridemia (HTG) severa ${ }^{41}$, destacando una frecuencia alélica muy baja $(0,003$ en controles y 0,016 en HTG).

El polimorfismo situado en la región intrónica del gen IVS3+476A (o SNP1) se ha asociado con el incremento de los niveles de TG y con el riego de desarrollar síndrome metabólico ${ }^{22}$.

Por otro lado, el polimorfismo -3G > A está localizado en la secuencia Kozak y aunque su estudio aislado no ha sido tan estudiado, su análisis se ha realizado formando parte de haplotipos. Dada su localización, inmediatamente antes del codón iniciador se ha sugerido que potencialmente podría alterar la traducción ${ }^{21}$.

\section{Principales interacciones gen-ambiente entre los polimorfismos de la apolipoproteína A5 y fenotipos intermedios de enfermedad cardiovascular}

Existen pocos estudios publicados que aborden las interacciones entre genes y patrones de dieta o consumo de alimentos. Así, mientras que en el trabajo de Pennachio y $\mathrm{col}^{6}$, no se encontró modificación de la lipidemia según el genotipo tras estudiar la dieta habitual de los participantes y después una intervención con dietas altas y bajas en grasa, estudios llevados a cabo en la cohorte Framingham pusieron de manifiesto algunas modulaciones de la concentración de TG y el peso en los portadores de la mutación por la dieta. Concretamente, el alelo -1131C estuvo asociado con mayores niveles de TG en ayunas sólo en personas que consumían altas cantidades de ácidos grasos poliinsaturados (AGPI) $(>6 \%)$ sin diferencia de sexo $^{42}$. También se encontró una interacción significativa entre el aporte de ácidos 
grasos monoinsaturados (AGM) y el polimorfismo del promotor $(-1131 \mathrm{~T} / \mathrm{C})$ de la $A P O A 5$ respecto al peso corporal, de forma que cuando la ingesta de AGM era alta, los portadores del alelo mutado (-1131C) tenían menor IMC que los homocigotos normales. Esta situación no ocurría con ingestas de AGM más bajas ${ }^{43}$. Este efecto de la perdida de peso (un 13,4\%) también se observó en hombres hiperlipémicos portadores del alelo $-1131 \mathrm{C}$ tras seguir una dieta con restricción energética durante tres meses y no en los homocigotos $\mathrm{TT}^{44}$. Asimismo, Hubacek y col $^{45}$ también evaluaron la respuesta al cambio de dieta según variaciones en el gen la $A P O A 5$ encontrando que los portadores del alelo Trp19 (S19W) diminuyeron los niveles de CT en más de $20 \%$.

\section{Asociaciones e interacciones entre los polimorfismos de la apolipoproteína A5 y fenotipos finales de ECV}

Por lo que respecta a la asociación entre la variación genética en el gen de la APOA5 y la aparición de fenotipos finales, cabe destacar que la mayor información presente a la literatura consultada corresponde a la cardiopatía isquémica. La Tabla 4 muestra los diferentes trabajos realizados al respecto, los polimorfismos estudiados, tipos de estudio y características de la muestra.

El polimorfismo que más frecuentemente ha sido asociado con la presencia de cardiopatía isquémica es - 1131T $>\mathrm{C}^{28,29,46-48}$. No obstante estos resultados no han sido replicados en todos los $\operatorname{casos}^{49,50}$. Por otro lado, el polimorfismo S19W no parece asociarse a la aparición de enfermedad coronaria $^{49,51}$. Sin embargo, el polimorfismo -1131 $\mathrm{T}>\mathrm{C}$ estuvo asociado al estenosis carotídea en el estudio Framingham ${ }^{52}$.

Otro fenotipo final cuya asociación con polimorfismos en APOA5 ha sido estudiado es el accidente cerebrovascular. Aunque la evidencia con respecto a este fenotipo final es más escasa y poco consistente, sugiere que el polimorfismo $-1131 \mathrm{~T}>\mathrm{C}$ se asocia a la presencia de enfermedad cerebrovascular ${ }^{33,54}$.

En cuanto al mecanismo por el cual polimorfismos en APOA5 pueden estar asociados a patología cardiovascular, si bien primariamente podría atribuirse a su impacto sobre la lipidemia, fundamentalmente sobre las concentraciones séricas de TG, modelos multivariante que incluyen este parámetro en la estimación de riesgo, parecen mostrar que esta asociación es independiente de los TG plasmáticos ${ }^{30,52}$. Una posible explicación es que estos polimorfismos se encuentren en desequilibrio de ligamiento con APOC3 como afirman Talmud $\mathrm{y} \mathrm{col}^{30}$. No obstante, otro estudio demostró la asociación de la variante $-1131 \mathrm{~T}>\mathrm{C}$ con cardiopatía, y descartó el desequilibrio de ligamiento con $A P O C 3$, sugiriendo que posiblemente $A P O A 5$ se relacionara con la cardiopatía a través de una modificación de la composición de las $\mathrm{HDL}^{28}$.

\section{Conclusiones}

El gen de la APOA5 es un gen candidato muy relevante en el estudio de las concentraciones plasmáticas de TG. Se han descrito más de 15 variantes en este gen aunque las más estudiadas han sido el -1131T > C y el S19W. Los estudios llevados a cabo en diferentes grupos étnicos, y a pesar de la distinta distribución genotípica, coinciden en la asociación de su alelo menos común con mayores concentraciones de TG, lo que sugiere que el genotipado de sus variantes podría tener utilidad en los pacientes con enfermedad cardiovacular. El polimorfismo $-1131 \mathrm{~T}>\mathrm{C}$ del promotor es el que más se ha asociado con el riesgo de cardiopatía isquémica y otros fenotipos finales cardiovasculares, aunque serían necesarios más estudios coincidentes en este sentido. En la actualidad, la genómica nutricional es una disciplina nueva y el estudio de las interacciones o modulaciones gen-dieta está cobrando mucha importancia. Sin embargo, son todavía escasos los estudios que se centren en este aspecto, y es necesario proseguir las investigaciones para su aplicación a la práctica clínica. El objetivo, por tanto es la integración del conocimiento no sólo bioquímico, genético sino de los factores ambientales para optar por un tratamiento más personalizado en la prevención de las enfermedades cardiovasculares.

Agradecimientos: Este trabajo ha sido parcialmente financiado por las ayudas CIBER06/03/035, PI070954 del Instituto de Salud Carlos III, y la Beca FPU (AP2007-04663) del Ministerio de Ciencia e Innovación, España. 


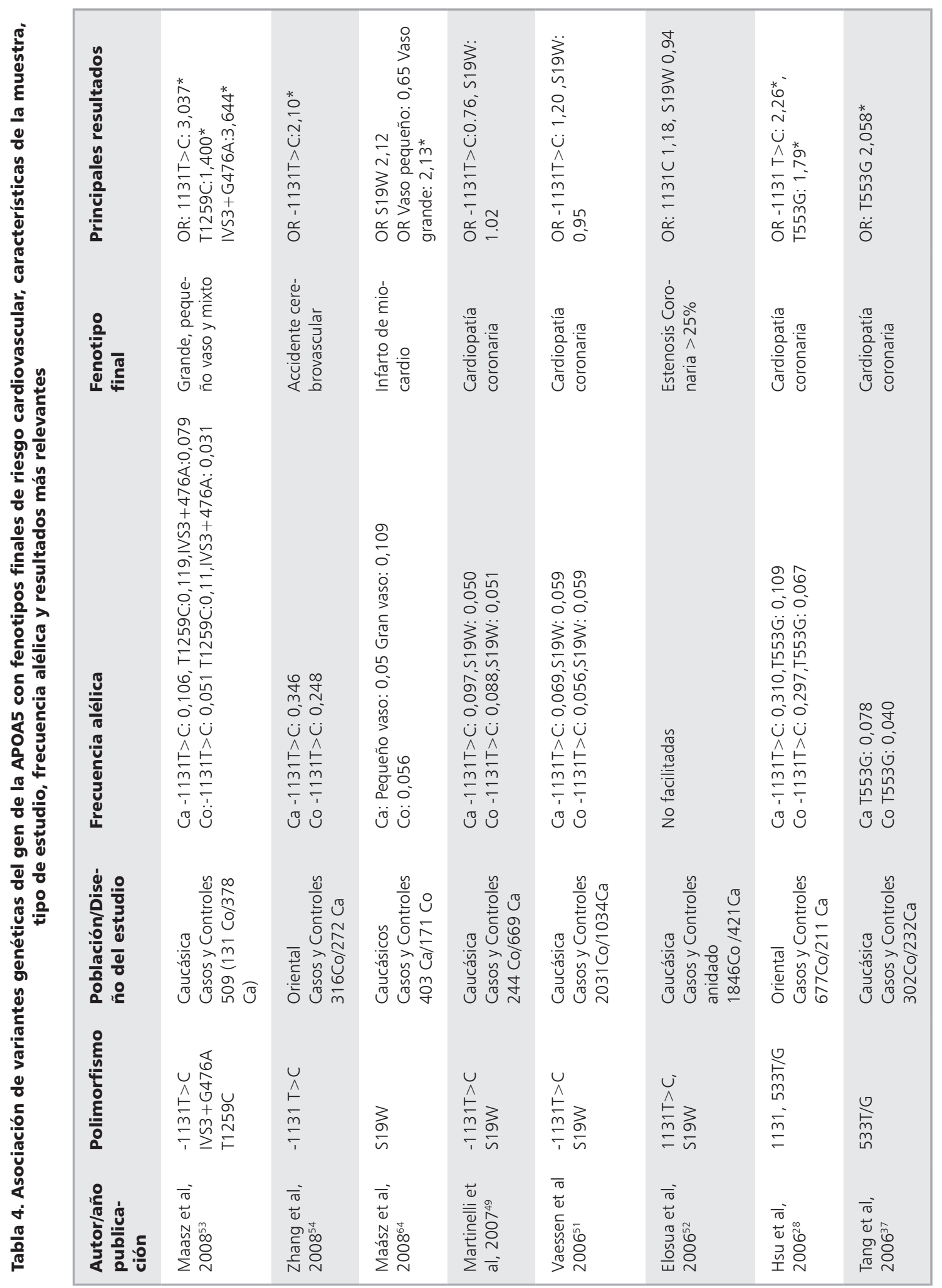




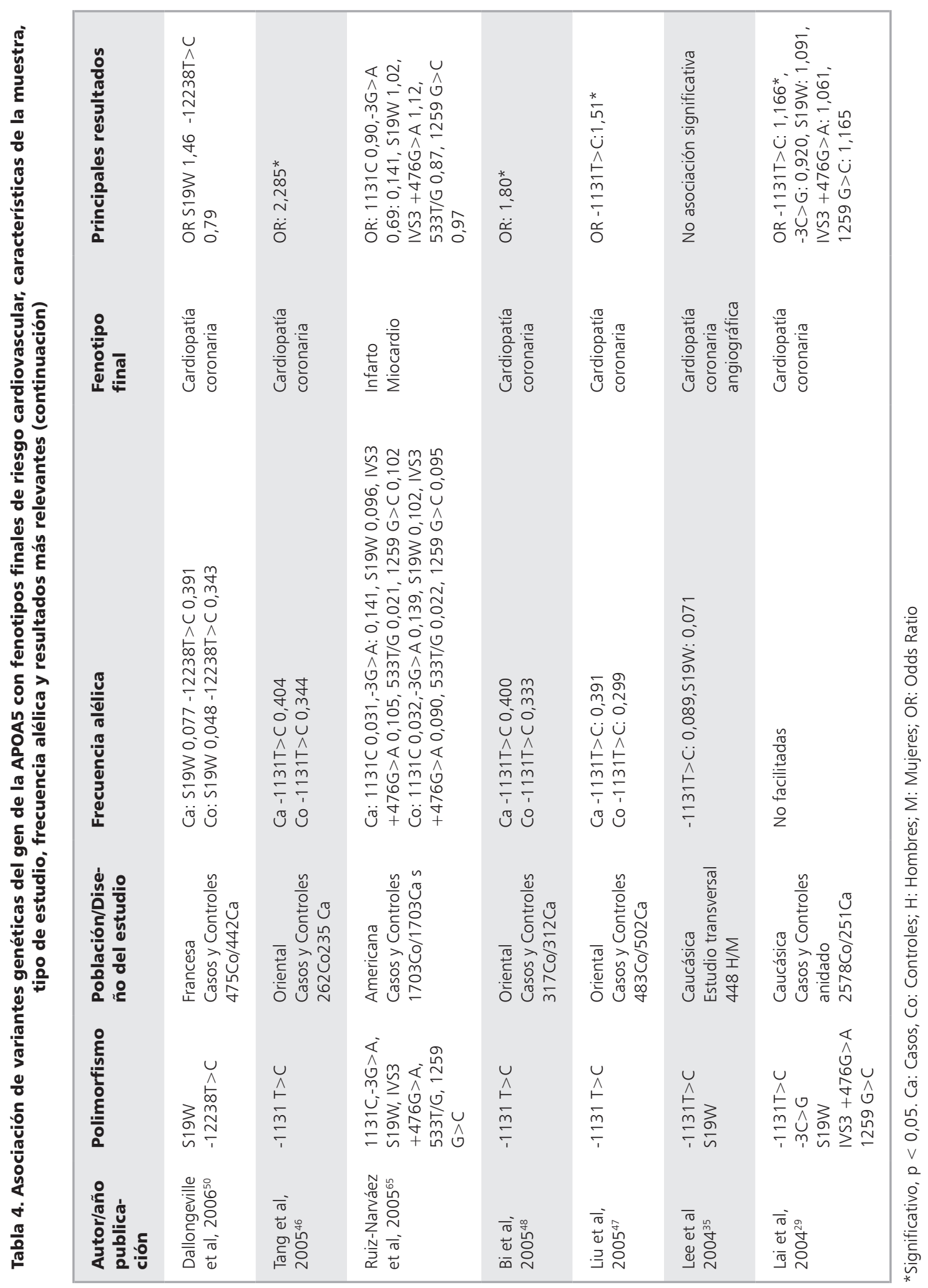




\section{Referencias}

1. Mcbride P. Triglycerides and risk for coronary artery disease. Curr Atheroscler Rep 2008; 10 (5): 386-90.

2. Ferns G, Keti V, Griffin B. Investigation and management of hypertriglyceridaemia. J Clin Pathol 2008; 61: 117483.

3. Malloy MJ, Kane JP. A risk factor for atherosclerosis: triglyceride-rich lipoproteins. Adv Intern Med 2001; 47: 111-36.

4. Eberly LE, Stamler J, Neaton JD; Multiple Risk Factor Intervention Trial Research Group. Relation of triglyceride levels, fasting and nonfasting, to fatal and nonfatal coronary heart disease. Arch Intern Med 2003; 12; 163 (9): 1077-83.

5. Abdel-Maksoud MF, Hokanson JE. The complex role of triglycerides in cardiovascular disease. Semin Vasc Med 2002; 2 (3): 325-33.

6. Pennacchio LA, Olivier M, Hubacek JA, Cohen JC, Cox DR, Fruchart JC, et al. An apolipoprotein influencing triglycerides in human and mice revealed by comparative sequencing. Science 2001; 294: 169-73.

7. Prieur X, Coste H, Rodríguez JC. The human apolipoprotein $\mathrm{AV}$ gene is regulated by peroxisome proliferatoractivated receptor-alpha and contains a novel farnesoid $\mathrm{X}$-activated receptor response element. J Biol Chem 2003; 278: 25468-80.

8. Vu-Dac N, Gervois P, Jakel H, Nowak M, Bauge E, Dehondt $\mathrm{H}$, et al. Apolipoprotein A5, a crucial determinant of plasma triglyceride level, is highly responsive to peroxisome proliferator-activated receptor alpha activators. J Biol Chem 2003; 278: 17982-5.

9. Genoux A, Dehondt H, Helleboid-Chapman A, Duhem C, Hum Dw, Martin G, et al. Transcriptional regulation of apolipoprotein A5 gene expression by the nuclear receptor ROR. Arterioscler Thromb Vasc Biol 2005; 25: 1186-92.

10. Jakel H, Nowak M, Moitrot E, Dehondt H, Hum Dw, Pennacchio LA, et al. The liver $\mathrm{X}$ receptor ligand T0901317 down-regulates APOA5 gene expression through activation of SREBP-1c. J Biol Chem 2004; 279: 45462-9.

11. Van Der Vliet HN, Sammelsm MG, Leegwater AC, Levels JH, Reitsma PH, Boers W, et al. Apolipoprotein A-V: a novel apolipoprotein associated with an early phase of liver regeneration. J Biol Chem 2001; 276: 44512-20.

12. O’Brien JP, Alborn WE, Sloan JH, Ulmer M, Boodhoo A, Knierman MD, et al. The Novel Apolipoprotein A5 Is Present in Human Serum, Is Associated with VLDL, HDL, and Chylomicrons, and Circulates at Very Low Concentrations Compared with Other Apolipoproteins.
Clin Chem 2005; 51: 351-9.

13. Fruchart-Najib J, Bauge E, Niculescu LS, Pham T, Thomas B, Rommens C, et al. Mechanism of triglyceride lowering in mice expressing human apolipoprotein A5. Biochem Biophys Res Commun 2004; 319: 397-404.

14. Alborn WE, Johnson MG, Prince MJ, Konrad RJ. Definitive N-terminal protein sequence and further characterization of the novel apolipoprotein A5 in human serum. Clin Chem 2006; 52 (3): 514-7.

15. Weinberg RB, Cook VR, Beckstead JA, Martin DD, Gallagher JW, Shelness GS, et al. Structure and interfacial properties of human apolipoprotein A-V. J Biol Chem 2003; 278 (36): 34438-44.

16. Beckstead JA, Wong K, Gupta V, Wan CP, Cook VR, Weinberg RB, et al. The $\mathrm{C}$ terminus of apolipoprotein A-V modulates lipid-binding activity. J Biol Chem 2007; 282 (21): 15484-9.

17. Schaap FG, Rensen PC, Voshol PJ, Vrins C, Van Der Vliet HN, Chamuleau RA, et al. ApoAV reduces plasma triglycerides by inhibiting very low density lipoproteintriglyceride (VLDL-TG) production and stimulating lipoprotein lipase-mediated VLDL-TG hydrolysis. J Biol Chem 2004; 279: 27941-7.

18. Merkel M, Loeffler B, Kluger M, Fabig N, Geppert G, Pennacchio LA, et al. Apolipoprotein AV accelerates plasma hydrolysis of triglyceride-rich lipoproteins by interaction with proteoglycan-bound lipoprotein lipase. J Biol Chem 2005; 280: 21553-60.

19. Kao JT, Wen HC, Chien KL, Hsu HC, Lin SW. A novel genetic variant in the apolipoprotein A5 gene is associated with hypertriglyceridemia. Hum Mol Genet 2003; 12: 2533-9.

20. Chien KL, Chen MF, Hsu HC, Su TC, Chang WT, Lee $\mathrm{CM}$, et al. Genetic association study of APOA1/C3/ A4/A5 gene cluster and haplotypes on triglyceride and HDL cholesterol in a community-based population. Clin Chim Acta 2008; 388 (1-2): 78-83.

21. Pennacchio LA, Olivier M, Hubacek JA, Krauss RM, Rubin EM, Cohen JC. Two independent apolipoprotein A5 haplotypes influence human plasma triglyceride levels 2002 15; 11 (24): 3031-8.

22. Kisfali P, Mohás M, Maasz A, Hadarits F, Markó L, Horvatovich $\mathrm{K}$, et al. Apolipoprotein A5 IVS3+476A allelic variant associates with increased trigliceride levels and confers risk for development of metabolic syndrome in Hungarians. Circ J 2008; 72 (1): 40-3.

23. Dorfmeister B, Cooper JA, Stephens JW, Ireland H, Hurel SJ, Humphries SE, et al. The effect of APOA5 and APOC3 variants on lipid parameters in European Whites, Indian Asians and Afro-Caribbeans with type 2 diabetes. Biochim Biophys Acta 2007; 1772 (3): 355-63. 
24. Martin M, Nicaud V, Humphries Se, Talmud PJ. Contribution of APOA5 gene variants to plasma triglyceride determination and to the response to both fat and glucose tolerance challenges. Biochim Biophys Acta 2003; 1637: 217-25.

25. Endo K, Yanagi H, Araki J, Hirano C, YamakawaKobayashi K, Tomura S. Association found between the promoter region polymorphism in the apolipoprotein $\mathrm{A}-\mathrm{V}$ gene and the serum triglyceride level in Japanese schoolchildren. Hum Genet 2002; 111: 570-2.

26. Lai CQ, Tai ES, Tan CE, Cutter J, Chew SK, Zhu YP, et al. The APOA5 locus is a strong determinant of plasma triglyceride concentrations across ethnic groups in Singapore 2003; 44 (12): 2365-73.

27. Chien KL, Fang WH, Wen HC, Lin HP, Lin YL, Lin SW, et al. APOA1/C3/A5 haplotype and risk of hypertriglyceridemia in Taiwanese. Clin Chim Acta 2008; 390 (1-2): 56-62.

28. Hsu LA, Ko YL, Chang CJ, Hu CF, Wu S, Teng MS, et al. Genetic variations of apolipoprotein A5 gene is associated with the risk of coronary artery disease among Chinese in Taiwan. Atherosclerosis 2006; 185 (1): 143-9.

29. Lai CQ, Demissie S, Cupples LA, Zhu Y, Adiconis X, Parnell LD, et al. Influence of the APOA5 locus on plasma triglyceride, lipoprotein subclasses, and CVD risk in the Framingham Heart Study. J Lipid Res 2004; 45 (11): 2096-105.

30. Talmud PJ, Hawe E, Martin S, Olivier M, Miller GJ, Rubin EM, et al. Relative contribution of variation within the APOC3/A4/A5 gene cluster in determining plasma triglycerides. Hum Mol Genet 2002; 15; 11 (24): 3039-4.

31. Chandak GR, Ward KJ, Yajnik CS, Pandit AN, Bavdekar A, Joglekar CV, et al. Triglyceride associated polymorphisms of the APOA5 gene have very different allele frequencies in Pune, India compared to Europeans. BMC Med Genet 2006; 10; 7-76.

32. Talmud PJ, Cooper JA, Hattori H, Miller IP, Miller GJ, Humphries SE. The apolipoprotein A-V genotype and plasma apolipoprotein $\mathrm{A}-\mathrm{V}$ and triglyceride levels: prospective risk of type 2 diabetes. Results from the Northwick Park Heart Study II. Diabetología 2006; 49 (10): 2337-40.

33. Qi L, Liu S, Rifai N, Hunter D, Hu FB. Associations of the apolipoprotein A1/C3/A4/A5 gene cluster with triglyceride and HDL cholesterol levels in women with type 2 diabetes. Atherosclerosis 2007; 192 (1): 204-10.

34. Grallert H, Sedlmeier Em, Huth C, Kolz M, Heid Im, Meisinger C, et al. APOA5 variants and metabolic syndrome in Caucasians. Lipid Res 2007; 48 (12): 2614-21.

35. Lee KW, Ayyobi AF, Frohlich JJ, Hill JS. APOA5 gene polymorphism modulates levels of triglyceride, HDL cholesterol and FERHDL but is not a risk factor for coronary artery disease. Atherosclerosis 2004; 176 (1): 165-72.

36. Hubácek JA, Adámková V, Ceska R, Poledne R, Horínek A, Vráblík M. New variants in the apolipoprotein AV gene in individuals with extreme triglyceride levels. Physiol Res 2004; 53 (2): 225-8.

37. Tang Y, Sun P, Guo D, Ferro A, Ji Y, Chen Q, et al. A genetic variant c.553G $>\mathrm{T}$ in the apolipoprotein A5 gene is associated with an increased risk of coronary artery disease and altered triglyceride levels in a Chinese population. Atherosclerosis 2006; 185 (2): 433-7.

38. Zhai G, Wen P, Guo L, Chen L. Association of APOA5 c.553G $>\mathrm{T}$ polymorphism with type 2 diabetes mellitus in a Chinese population. Clin Chem Lab Med 2006; 44 (11): 1313-6.

39. Matsunaga A, Arishima $H$, Niimura $H$, Zhang B, Uehara $\mathrm{Y}$, Ohwaki K, et al. Strong linkage disequilibrium and association of $-1131 \mathrm{~T}>\mathrm{C}$ and c.553G $>$ T polymorphisms of the apolipoprotein A5 gene with hypertriglyceridemia in a Japanese population. Circ J 2007; 71 (5): 746-52.

40. Hubacek JA, Skodová Z, Adámková V, Lánská V, Poledne R. Sex-specific effect of APOAV variant (Val153 > Met) on plasma levels of high-density lipoprotein cholesterol. Metabolism 2005; 54 (12): 1632-5.

41. Hubacek JA, Wang WW, Skodová Z, Adámková V, Vráblík M, Horínek A, et al. APOA5 Ala315>Val, identified in patients with severe hypertriglyceridemia, is a common mutation with no major effects on plasma lipid levels. Clin Chem Lab Med 2008; 46 (6): 773-7.

42. Lai CQ, Corella D, Demissie S, Cupples La, Adiconis X, Zhu Y, et al. Dietary intake of n-6 fatty acids modulates effect of apolipoprotein A5 gene on plasma fasting triglycerides, remnant lipoprotein concentrations, and lipoprotein particle size: the Framingham Heart Study. Circulation 2006; 113 (17): 2062-70.

43. Corella D, Lai CQ, Demissie S, Cupples LA, Manning AK, Tucker KL, et al. APOA5 gene variation modulates the effects of dietary fat intake on body mass index and obesity risk in the Framingham Heart Study. J Mol Med 2007; 85 (2): 119-28.

44. Aberle J, Evans D, Beil FU, Seedorf U. A polymorphism in the apolipoprotein A5 gene is associated with weight loss after short-term diet. Clin Genet 2005; 68 (2): 152-4.

45. Hubacek JA, Skodová Z, Adámková V, Lánská V, Pitha J. APOA5 variant Ser19Trp influences a decrease of the total cholesterol in a male 8 year cohort. Clin Biochem 2006; 39 (2): 133-6.

46. Tang YB, Sun P, Guo DP, Li XY, Chen Q, Fan LM. Association between apolipoprotein A5 -1131T > C polymorphism and susceptibility of coronary artery disease 
in Chinese. Zhonghua Yi Xue Yi Chuan Xue Za Zhi 2005; 22 (3): 281-3.

47. Liu H, Zhang S, Lin J, Li H, Huang A, Xiao C, et al. Association between DNA variant sites in the apolipoprotein A5 gene and coronary heart disease in Chinese. Metabolism 2005; 54 (5): 568-72.

48. Bi N, Yan SK, Li GP, Yin ZN, Xue H, Wu G, et al. Polymorphsims in the apolipoprotein A5 gene and apolipoprotein $\mathrm{C} 3$ gene in patients with coronary artery disease Zhonghua Xin Xue Guan Bing Za Zhi 2005; 33 (2): 116-21.

49. Martinelli N, Trabetti E, Bassi A, Girelli D, Friso S, Pizzolo $\mathrm{F}$, et al. The $-1131 \mathrm{~T}>\mathrm{C}$ and S19W APOA5 gene polymorphisms are associated with high levels of triglycerides and apolipoprotein C-III, but not with coronary artery disease: an angiographic study. Atherosclerosis. 2007; 191 (2): 409-17.

50. Dallongeville J, Cottel D, Montaye M, Codron V, Amouyel P, Helbecque N. Impact of APOA5/A4/C3 genetic polymorphisms on lipid variables and cardiovascular disease risk in French men. Int J Cardiol 2006; 106 (2): 152-6.

51. Vaessen SF, Schaap FG, Kuivenhoven JA, Groen AK, Hutten BA, Boekholdt SM, et al. Apolipoprotein A-V, triglycerides and risk of coronary artery disease: the prospective Epic-Norfolk Population Study. J Lipid Res 2006; 47 (9): 2064-70.

52. Elosua R, Ordovas JM, Cupples LA, Lai CQ, Demissie S, Fox CS, et al. Variants at the APOA5 locus, association with carotid atherosclerosis, and modification by obesity: the Framingham Study. J Lipid Res 2006; 47 (5): 990-6.

53. Maasz A, Kisfali P, Jaromi L, Horvatovich K, Szolnoki Z, Csongei V, et al. Apolipoprotein A5 gene IVS3+G476A allelic variant confers susceptibility for development of ischemic stroke. Circ J 2008; 72 (7): 1065-70.

54. Zhang K, Qiu F, Li L, Gu GY, Tao Y, Wang L, et al. The associated study on apolipoprotein A5 gene polymorphisms with carotid artherosclerosis in patients with cerebral infartion. Zhonghua Yi Xue Yi Chuan Xue Za Zhi 2008; 25 (3): 284-8.

55. Komurcu-Bayrak E, Onat A, Poda M, Humphries Se, Palmen J, Guclu F, et al. Gender-modulated impact of apolipoprotein A5 gene (APOA5) -1131T > C and c.56C > G polymorphisms on lipids, dyslipidemia and metabolic syndrome in Turkish adults. Clin Chem Lab Med 2008; 46 (6): 778-84.

56. Jiang YD, Yen CJ, Chou WL, Kuo SS, Lee KC, Chiu KC, et al. Interaction of the G182C polymorphism in the APOA5 gene and fasting plasma glucose on plasma triglycerides in Type 2 diabetic subjects. Diabet Med 2005;
22 (12): 1690-5.

57. Bi N, Yan SK, Li GP, Yin ZN, Chen BS. A single nucleotide polymorphism $-1131 \mathrm{~T}>\mathrm{C}$ in the apolipoprotein A5 gene is associated with an increased risk of coronary artery disease and alters triglyceride metabolism in Chinese. Mol Genet Metab 2004; 83 (3): 280-6.

58. Moreno-Luna R, Pérez-Jiménez F, Marín C, PérezMartínez P, Gómez P, Jiménez-Gómez Y, et al. Two independent apolipoprotein A5 haplotypes modulate postprandial lipoprotein metabolism in a healthy Caucasian population. J Clin Endocrinol Metab 2007; 92 (6): 2280-5.

59. Kahri J, Fruchart-Najib J, Matikainen N, Fruchart JC, Vakkilainen J, Taskinen MR. The increase of apolipoprotein $\mathrm{A}-\mathrm{V}$ during postprandial lipemia parallels the response of triglyceride-rich lipoproteins in type 2 diabetes: no relationship between apoA- $\mathrm{V}$ and postheparin plasma lipolytic activity. Diabetes Care 2007; 30 (8): 2083-5.

60. Kim JY, Kim OY, Koh SJ, Jang Y, Yun SS, Ordovas JM, et al. Comparison of low-fat meal and high-fat meal on postprandial lipemic response in non-obese men according to the -1131T $>$ C polymorphism of the apolipoprotein A5 (APOA5) gene (randomized cross-over design). J Am Coll Nutr 2006; 25 (4): 340-7.

61. Moreno R, Pérez-Jiménez F, Marín C, Moreno JA, Gómez P, Bellido C, et al. A single nucleotide polymorphism of the apolipoprotein A-V gene -1131T > C modulates postprandial lipoprotein metabolism. Atherosclerosis 2006; 189 (1): 163-8.

62. Pruneta-Deloche V, Ponsin G, Groisne L, Fruchart-Najib J, Lagarde M, Moulin P. Postprandial increase of plasma apoAV concentrations in Type 2 diabetic patients. Atherosclerosis 2005; 181 (2): 403-5.

63. Jang Y, Kim JY, Kim OY, Lee JE, Cho H, Ordovas JM, et al. The -1131T-->C polymorphism in the apolipoprotein A5 gene is associated with postprandial hypertriacylglycerolemia; elevated small, dense LDL concentrations; and oxidative stress in nonobese Korean men. Am J Clin Nutr 200; 80 (4): 832-40.

64. Maász A, Kisfali P, Szolnoki Z, Hadarits F, Melegh B. Apolipoprotein A5 gene C56G variant confers risk for the development of large-vessel associated ischemic stroke. J Neurol 2008; 255 (5): 649-54.

65. Ruiz-Narváez EA, Yang Y, Nakanishi Y, Kirchdorfer J, Campos H. APOC3/A5 haplotypes, lipid levels, and risk of myocardial infarction in the Central Valley of Costa Rica 2005; 46 (12): 2605-13.

66. Doyle PS, Bibette J, Bancaud A, Viovy JL. Self-assembled magnetic matrices for DNA separation chips. Science. 2002; 22; 295 (5563): 2237. 